\title{
Research on Optimization of Logistics Transportation Mode Based on Multimodal Transportation
}

\author{
Xiangfeng Zhou, Juan Du* \\ School of Transportation and Logistics \\ East China Jiaotong University \\ Nanchang, China \\ 406671690@qq.com,1415823746@qq.com*
}

\begin{abstract}
Multimodal transport is an efficient way of transportation organization. It can make full use of transportation resources, promote the rational division of labor of various modes of transportation, reduce transportation cost and logistics cost.This paper links the whole vehicle logistics with multimodal transport, and builds a multi-modal transport path optimization model with time windows. The total logistics cost is currently the lowest. Considering transportation cost, replacement cost, storage cost or penalty cost and risk cost that may occur in transportation and reloading, the genetic algorithm is used to solve it, which is realized in Matlab and combined with case analysis.
\end{abstract}

Keywords-Multimodal transport; Time window; Path optimization; Genetic algorithm

\section{INTRODUCTION}

With the rapid growth of China's economy, the logistics industry has also developed rapidly, and the role of logistics in social and economic development has become increasingly prominent. For multimodal transport problems, domestic and foreign scholars have carried out a lot of research on it, and Hei Xiuling [1] proposed the opportunity constrained programming model of multimodal transport. Yi Yunfei et al [2] used the minimization of transportation cost and transshipment cost as the objective function to construct a mathematical programming model for multimodal transport path selection. Ekki.D.K[3] proposed a 0-1 planning model for multimodal transport hub location. CHANG.T[4] constructed a multiobjective optimization model for multi-modal transport path selection considering multi-commodity flow. The model considers time window constraints, transportation mode selection, and transportation cost. The above studies are carried out separately for these two types of problems. The study of vehicle paths with time windows is mainly based on soft time window constraints, while the multi-modal transport research objects are more directed at containers, but the two studies are less comprehensive, and there are also few studies on multimodal transport of vehicle logistics. In this paper, the vehicle routing problem with time window is introduced into the theory of multimodal transport. For the logistics cost problem of the whole vehicle, the multi-modal transport line optimization model with time window is established.

\section{MULTIMODAL TRANSPORT MODEL CONSTRUCTION}

\section{A. Model Hypothesis}

- Simplify cities into mass points, taking into account the impact of city size on transportation and replacement costs.

- Only one transportation mode is used between every two adjacent nodes, and the reloading must be carried out in the node city.

- The risks and losses due to natural factors are difficult to measure, so the effects of natural factors are not considered.

\section{B. Model Building}

$$
Z=\min \left\{\begin{array}{l}
\left\{\sum_{i \in N} \sum_{j \in N} \sum_{k \in M}\left[q(i)\left(c_{i j}^{k}+e_{i j}^{k}\right) r_{i j}^{k}\right] x_{i j}^{k}\right\}+ \\
\left\{\sum_{i \in N} \sum_{l \in M} \sum_{k \in M}\left[q(i)\left(p_{i}^{k l}+o_{i}^{k l}\right)\right] y_{i}^{k l}\right\}+F(t)
\end{array}\right\}
$$

The constraints are:

$$
\begin{gathered}
\sum_{i \in N} \sum_{j \in N} \sum_{k \in M} x_{i j}^{k} \omega_{i j}^{k}+\sum_{i \in N} \sum_{j \in N} \sum_{k \in M} q(i) y_{i}^{k l} u_{i}^{k l}=t \\
F(t)= \begin{cases}\left(a_{i}-t\right) S_{1} & t<a_{i} \\
0 & a_{i} \leq t \leq b_{i} \\
\left(t-b_{i}\right) S_{2} & b_{i}<t\end{cases} \\
x_{i j}^{k}= \begin{cases}0 & i=0 \\
1 & i \neq 0\end{cases}
\end{gathered}
$$




$$
\mathrm{y}_{i}^{k l}= \begin{cases}0 & i=0 \\ 1 & i \neq 0\end{cases}
$$

When i, j is determined, $\sum_{k \in M} x_{i j}^{k}=1$

When i is determined, $\sum_{l \in M} \sum_{k \in M} y_{i}^{k l}=1$

$$
\begin{gathered}
\sum_{i \in N} \sum_{j \in N} v_{i j} x_{i j}^{k} \geq q(i), k \in M \\
\sum_{i \in N} \sum_{j \in N} v_{i j} x_{i j}^{k} \leq V_{i j}, k \in M
\end{gathered}
$$

In the formula: $Z$ is the total cost of logistics, 10,000 yuan(1); $Q(i)$ is the total demand, 10,000 units; $q(i)$ is the node $i$ demand, 10,000 units; $N$ is the node city collection(1)(2); $M$ is the transportation mode collection, where $m \in M$ (6)(7), $m=1,2,3$ (1-Highway;2 - Railway; 3waterway; $v_{i j}$ is the actual traffic from point to point $j ; V_{i j}$ is the maximum traffic of the route from point $i$ to point $j(8)(9)$; $C_{i j}^{k}$ is the unit that is transported to point $j$ by point $k$ at point $i$ (1)(4), transportation cost, 10,000 yuan / $(100 \mathrm{~km} \cdot 10,000$ units $) ; e_{i j}^{k}$ is the risk cost when transporting to $j$ point by point $k$ at point $i$ (1)(4), 10,000 yuan / $(100 \mathrm{~km} \cdot 10,000$ units $) ; r_{i j}{ }^{k}$ is the transport distance transported to point $j$ by $k$ at point $i, 100 \mathrm{~km}(1)(4) ; \omega_{i j}^{k}$ is the transportation time of transportation to point $j$ by point $k$ at point $i, \mathrm{~h}(2)(4) ; u_{i}^{k l}$ is the reloading time of changing the transportation mode from $k$ mode to $l$ mode at point $i, \mathrm{~h} / 10,000$ $\operatorname{sets}(2)(5) ; p_{i}^{k l}$ is the replacement cost generated by changing the transportation mode from $k$ mode to $l$ mode at point $i$, 10,000 yuan $/ 10,000$ units(1)(5); $O_{i}^{k l}$ is the risk cost of changing the transportation mode from $k$ mode to $l$ mode at point $i, 10,000$ Yuan $/ 10,000$ units(1)(5); $F(t)$ is the penalty cost for violation of time requirements, 10,000 yuan(1)(3); $t$ is the total time to transport the goods from the starting point to the destination, $\mathrm{h}(3) ;\left[a_{i}, b_{i}\right]$ is the time window of the arrival point, $\mathrm{h}(3) ; S_{1}$ is the cost of warehousing arrive early(3); $S_{2}$ to punish the cost of delayed arrival yuan(3)[5] .

\section{SOLUTION AND CASE ANALYSIS}

\section{A. Solution Step}

The genetic algorithm is used to solve the model mainly in six steps.

- Code Design The binary-coded model is used to determine whether or not to use the mode of transport between nodes and nodes, and whether to replace the code into chromosomes.

- Determination of population size The number of populations is A (even), and each iteration generates A [2(n$2)+1$ ]-dimensional vectors, and finds the optimal solution from these A vectors and records them. Through multiple iterations of selection, intersection, and variation, until the global optimal solution is generated, a random method is used to generate the initial population.

- Design of fitness function The fitness function is the total $\operatorname{cost} Z$ of each scheme. The higher fitness indicates that the total cost is lower, while the lower the fitness is higher.

- Select an action In the model, the total cost of the route is low, but the fitness is large. Therefore, the total cost $Z$ is counted down by $1 / Z$, and the fitness $p_{1}$ of each route is $p_{1}=\left(1 / Z_{1}\right) / \operatorname{sum}(1 / Z)$.

- Cross operation The population A vector is equally divided into upper and lower $\mathrm{A} / 2$ strips, and the upper $X$ strip vector is paired with the lower $X$ strip. The crossover starts from the first vector until the end of the A/2 vector. An evenly distributed pseudo-random number $\operatorname{rand}(0-1)$ is set as a basis, and if rand is less than the set crossover probability, the crossover operation is entered.

- Mutation operation Start with the first vector and until the end of the A vector. The mutation operation is also divided into two parts, namely n-1 dimension and n-2 dimension before each vector, and the position of the mutation is randomly selected in the first n-1 dimension and the latter n-2 dimension. For the former $n-1$ dimensional variation, the value of the mutation is again selected from 1 to $\mathrm{m}$ outside the original value; for the latter $n-2$ dimensional variation, since it is a $0-1$ programming model, the variation is 0 to 1 and 1 mutation is used. into 0 .

Finally, it is judged whether the iteration termination condition is reached. If the group update iteration is otherwise performed, jump back to step 3 to repeat the operation, and the loop iteration until the output meets the condition[6].

\section{B. Case Analysis}

At present, $\mathrm{H}$ company has a total of $6 \mathrm{VDC}$ (vehicle distribution center) and 10 large VSC (vehicle storage center).Take the Yangtze River business of $\mathrm{H}$ Company as an example. The company has three VDCs along the Yangtze River. For the convenience of calculation, it is called Shanghai VDC(a). There are four VSCs in Nanjing (b), Wuhan (c), Chongqing (d) and Deyang. (e). The research on H-type multimodal transport of $\mathrm{H}$ company is mainly from Shanghai VDC to Nanjing VSC, Wuhan VSC, Chongqing VSC and Deyang 
VSC nodes. Each node has three modes: water transport, railway and highway.

Inquire about the transportation distance of each node in various ways, and calculate the transportation time according to the average speed of each transportation mode (the average speed of railway, automobile and inland vessels is 80, 100, $20 \mathrm{~km} / \mathrm{h}$ respectively), as shown in Table I. In order to facilitate the research, it is assumed that there is no difference in the unit cost and replacement cost of each transportation mode in different cities. According to the optimal path and distance of each transportation mode, as well as the operating cost, management cost, fuel cost, etc., the transportation cost of each mode can be calculated. Table II. The reloading cost mainly comes from the loading and unloading equipment and manpower. According to the maintenance cost, fuel cost, equipment depreciation cost and labor cost of the loading and unloading equipment, the replacement cost can be obtained. The reloading time is calculated by the loading and unloading efficiency of each mode, and the replacement cost is available. And the reloading time is only related to the two modes of transportation before and after the node; the risk cost is estimated according to the loss and damage, which is shown in Table III. The demand for each VSC of the G appliance brand is shown in Table IV. The time window set is shown in Table $\mathrm{V}$. If it arrives in advance, set the storage cost to 30,000 yuan/(10,000 units/hour). If the delay is reached, set the penalty cost to 30 . Ten thousand yuan / (10,000 units $\cdot$ hours $)$.

TABLE I. THREE WAYS DISTANCE AND TIME

\begin{tabular}{|l|l|l|l|l|l|l|}
\hline \multirow{2}{*}{$\begin{array}{c}\text { Transfer } \\
\text { point }\end{array}$} & \multicolumn{2}{|c|}{ Road } & \multicolumn{2}{c|}{ railway } & \multicolumn{2}{c|}{ waterway } \\
\cline { 2 - 7 } & $\begin{array}{c}\text { distanc } \\
\boldsymbol{e}\end{array}$ & time & $\begin{array}{c}\text { distanc } \\
\boldsymbol{e}\end{array}$ & time & $\begin{array}{c}\text { distan } \\
\boldsymbol{c e}\end{array}$ & time \\
\hline SH-NJ & 3.1 & 3 & 3.0 & 4 & 4.1 & 21 \\
\hline NJ-WH & 5.4 & 5 & 5.2 & 6 & 7.1 & 35 \\
\hline WH-CQ & 8.7 & 8 & 8.8 & 11 & 12.8 & 64 \\
\hline CQ-DY & 3.8 & 4 & 3.7 & 5 & 5.6 & 28 \\
\hline
\end{tabular}

TABLE II. THREE MODES OF TRANSPORTATION COST AND RISK COST

\begin{tabular}{|l|l|l|}
\hline \multicolumn{1}{|c|}{ Mode of transport } & transportation cost & Risk cost \\
\hline Road & 30 & 24 \\
\hline railway & 23 & 20 \\
\hline waterway & 12 & 12 \\
\hline \multicolumn{2}{|c|}{ b. Unit: 10,000 yuan $100 \mathrm{~km} / 10,000$ units }
\end{tabular}

TABLE III. THREE WAYS TO CHANGE THE TIME AND CHANGE THE COST

\begin{tabular}{|l|l|l|l|l|l|l|}
\hline \multirow{2}{*}{ transport } & \multicolumn{2}{|c|}{ Road } & \multicolumn{2}{c|}{ railway } & \multicolumn{2}{c|}{ waterway } \\
\cline { 2 - 7 } & time & cost & time & cost & time & cost \\
\hline Road & 0 & 0 & 10 & 80.4 & 20 & 180.8 \\
\hline railway & 10 & 81.4 & 0 & 0 & 30 & 240.1 \\
\hline waterway & 20 & 182.7 & 30 & 240.1 & 0 & 0 \\
\hline
\end{tabular}

\footnotetext{
c. Note: The unit of reloading time: $h / 10,000$ units; the unit cost of reloading: 10,000 yuan/10,000 units.
}

TABLE IV. DEMAND FOR THE CITY WHERE THE G ELECTRICAL BRAND VSC IS LOCATED IN 2017

\begin{tabular}{|c|l|l|l|l|}
\hline Demand & NJ & WH & CQ & DY \\
\hline 10,000 units & 0.5 & 0.3 & 0.2 & 0.3 \\
\hline
\end{tabular}

TABLE V. TIME WINDOW BETWEEN EACH NODE CITY

\begin{tabular}{|l|l|l|c|c|}
\hline \multicolumn{1}{|c|}{ path } & \multicolumn{1}{|c|}{ SH-NJ } & \multicolumn{1}{c|}{ SH-WH } & SH-CQ & SH-DY \\
\hline $\mathrm{h}$ & $(10,20)$ & $(40,50)$ & $(80,100)$ & $(100,120)$ \\
\hline
\end{tabular}

The program is solved by Matlab, and the relevant parameters of the genetic algorithm are set as follows: the population size is 30 , the mutation probability is 0.04 , the crossover probability is 0.5 , the maximum number of iterations is 100 , and the algorithm converges in 28 cycles, that is, the optimal combination of the problem for the 2,2,3,1, that is, Shanghai-Nanjing,Nanjing-Wuhan, Wuhan-Chongqing,

Chongqing-Deyang, respectively, the railway, waterway, and road transportation modes are adopted respectively. At this time, the total logistics cost is the lowest $=13.527$ million, which is The total cost of pure road transportation has saved $1,196,900$ yuan, which fully reflects the advantages of using three modes of transportation. It can be seen that the model and algorithm have significant optimization effects.

\section{CONCLUSION}

This paper comprehensively considers the requirements of logistics cost and timeliness, combines the vehicle path with time window and multimodal transport problem, and applies it to vehicle logistics, which fully reflects the advantages of the three modes of transportation, thus reducing the total logistics cost. Establish a multi-modal multi-modal transport 0-1 integer programming model with minimum total logistics cost as the target, including transportation costs, replacement costs, risk costs incurred during transportation and replacement, and early arrival or delay. The warehousing cost or penalty cost of arrival is solved by genetic algorithm, which solves the path optimization problem in the logistics distribution of the whole vehicle, and the example is verified. The results show that the model and algorithm are feasible and practical in the multimodal transport of vehicle logistics.

\section{REFERENCES}

[1] X.L. Hei, Research on multi-modal transportation optimization of automobiles, Nanjing: Southeast University, 2015(12), pp. 132-145.

[2] Y.F. Yi and W.Y. Dong. "Improved ito algorithm for solving vehicle routing problem with soft time window and its convergence analysis," Electronic Journal, vol. 4, pp. 658-664, 2015.

[3] D.K. Ekki, "Distance and time in intermodalgoods transport networks in Europe: a generic approach," Transportation Research Part A: Policy and Practice, vol. 42(7), pp. 973-993, 2008.

[4] T. Chang, "Best routes selection in international in-termodal net works, " Computers \& Operations Research, vol. 35(9), pp. 2877-2891, 2008 .

[5] G.M. Qin, Study on the choice of urban rail transit connection method and the attraction range of passenger flow, Harbin: Harbin Institute of Technology, 2010(7), pp. 176-178.

[6] T. Wang and G. Wang, "Research on a Combined Optimization Model of Multimodal Transport Network Transportation Mode," China Engineering Science, vol. 7(10), pp. 432-445, 2015. 